\title{
Induced and Natural Epigenetic Variation
}

\author{
H. Yi, N.C. Riddle* , T.L. STOKEs ${ }^{\dagger}$, H.-R. Woo, AND E.J. RichaRdS \\ Department of Biology, Washington University, St. Louis, Missouri 63130
}

The molecular nature of the inherited variation that underlies morphological and biochemical diversity generally is assumed to be genetic. In other words, the range of form among individuals and species is caused by changes in primary nucleotide sequence. This view has been cemented by countless studies defining the molecular genetic basis of important changes driving alternative developmental programs or characterizing evolutionary lineages. However, it is becoming increasingly evident that nongenetic alterations can have a striking impact on the morphology and physiology of cells and organisms. Such epigenetic alterations, which do not change nucleotide sequence but do affect the expression of this genetic information, can involve diverse mechanisms, including chromatin structural modifications and steady-state regulatory networks. A growing list of epigenetic alterations have been induced and studied in laboratory settings, and a few examples of spontaneous epigenetic alleles in a natural context have been reported.

Is epigenetic variation an important component of the raw material acted on by artificial and natural selection? The view that epigenetic variation plays a role in evolutionary change has been challenged, in part, because of the perceived similarity between epigenetic variation and Lamarckian evolution. The parallels between this widely discredited view of evolution by the inheritance of acquired characters and epigenetic inheritance stems from the possibility that environmentally induced adaptive variation in an individual can be passed on to future generations. While there is nothing controversial about the notion that environmental conditions (e.g., mutagens) can influence the generation of heritable variation, conventional neo-Darwinian models assume that variation is random and not adapted to the particular inducing environment. However, epigenetic variation is frequently nonrandom and might lead to inheritance of gene expression programs that are induced by the environment.

Significant objections based on mechanism also are leveled against the notion that epigenetic variation could fuel evolution (Maynard Smith 1990). The first objection is that epigenetic variation cannot be important in transmission genetics or evolution because such variation is confined to a single generation as a result of an erasure/resetting event during the organism's life cycle. This view

\footnotetext{
${ }^{*}$ Present address: Division of Biological Sciences, University of Missouri-Columbia, Columbia, Missouri 65211.

${ }^{\dagger}$ Present address: New York University, Department of Biology, New York, New York 10003.
}

incorporates a central aspect of the original definition of epigenetics put forward by Waddington (1939), a definition we would currently equate with developmental mechanisms of differential gene expression, signal transduction, and morphogenesis. The more specialized usage of the term epigenetics focuses on the acquisition, elaboration, and inheritance of biochemical marks that are superimposed on genetic information (Russo et al. 1996; Rice and Allis 2001; Richards and Elgin 2002). These marks need not be confined to a single generation. For example, whereas a widespread erasure and resetting of cytosine methylation patterns occurs in early mammalian embryogenesis (Reik et al. 2001; Santos et al. 2002), DNA methylation patterns can be inherited across generations in many organisms, including mammals (Silva and White 1988; Morgan et al. 1999; Rakyan et al. 2003), because some cytosine methylation sites can escape erasure (Kakutani et al. 1999; Macleod et al. 1999).

Even if it is conceded that epigenetic information can be inherited between organismal generations, including between sexual generations traversing meiosis, another serious objection arises - metastability. Indeed, instability and reversibility are among the features that continue to draw researchers' attention to classic epigenetic phenomena, such as nucleolar dominance (Pikaard 2000), paramutation (Chandler and Stam 2004), transposon changes in phase (McClintock 1952), and X-chromosome inactivation (Plath et al. 2002). It is argued that epigenetic changes cannot be relevant in an evolutionary context, or even in a breeding context, because inherited epigenetic variation is too unstable. Although metastability is a frequent feature of epigenetic variation, stable epigenetic alterations also exist that can be inherited with a high degree of fidelity mimicking that of traditional genetic Mendelian factors (Holliday and Ho 1990).

To learn more about the characteristics of epigenetic variation and the mechanisms that underlie this variation, our lab has been pursuing two different approaches using the flowering plant Arabidopsis thaliana. These two approaches will be summarized below in brief, and we will draw connections between our findings and some of the related work from other groups.

\section{INDUCED EPIGENETIC VARIATION}

The first approach grew out of our work on Arabidopsis mutants with dramatically reduced cytosine methylation. These mutants include plants deficient in either the 
DDM1 SWI2/SNF2 nucleosome remodeling factor (Vongs et al. 1993; Jeddeloh et al. 1999) or the Dnmt1class cytosine-DNA-methyltransferase MET1 (Kankel et al. 2003). Given the prevailing view at the time (early 1990s), it was expected that such mutants should be severely developmentally compromised. This view was solidified by the landmark paper published in 1992 by Li et al. (1992) demonstrating that Dnmt1 cytosine-DNAmethyltransferase knockout mice were inviable. In contrast, Arabidopsis DNA hypomethylation mutants did not exhibit significant developmental abnormalities (Vongs et al. 1993). Still more puzzling was the stochastic occurrence of morphologically aberrant individuals in $d d m 1$ mutant lines that had been propagated through several generations by self-pollination (Kakutani et al. 1996). Indeed, progressively more severe phenotypes were seen in inbred $d d m 1$ lines as the number of self-pollinations increased. Subsequent genetic analysis demonstrated that the morphological defects were caused by the accumulation of stable alterations at a limited number of genomic loci. Our lab and others have characterized a handful of these alterations during the past several years. One of the best-understood alterations induced by $d d m 1$ or met 1 is hypomethylated epigenetic alleles (Kakutani 1997; Kankel et al. 2003) of the FWA locus that lead to a delay in flowering time associated with ectopic expression of the putative transcription factor encoded by the locus (Soppe et al. 2000).

Our lab has focused on another $d d m 1$-induced alteration, called bal, which causes dwarfing and twisted leaf morphology (Stokes et al. 2002). This developmental syndrome stems from an alteration mapping to a complex $\sim 90-\mathrm{kb}$ cluster of NBS-LRR class (nucleotide binding site-leucine rich repeat) Resistance or $R$ genes and transposable elements on the lower arm of chromosome 4 (Fig. $1)$. These $R$ genes encode signal transduction components that act early in pathogen recognition and defense pathways (Dangl and Jones 2001). The bal defect causes an overexpression of $A t 4 g 16890$, the most highly expressed gene in the cluster in wild-type plants (Stokes et al. 2002). Overexpression of $\mathrm{At} 4 \mathrm{~g} 16890$ in transgenic plants recapitulates the phenotype. This overexpression leads to constitutive signaling through the salicylic acid (SA) pathway, which induces pathogenesis-related (PR) proteins, eventually leading to dwarfing and twisted leaf morphology. The sequence of $A t 4 g 16890$ in the bal variant is identical to that seen in wild-type plants (strain Columbia), suggesting that the genomic change causing the bal syndrome is epigenetic or resides within the $R$-gene cluster at some distance from At4g16890, or possibly both.

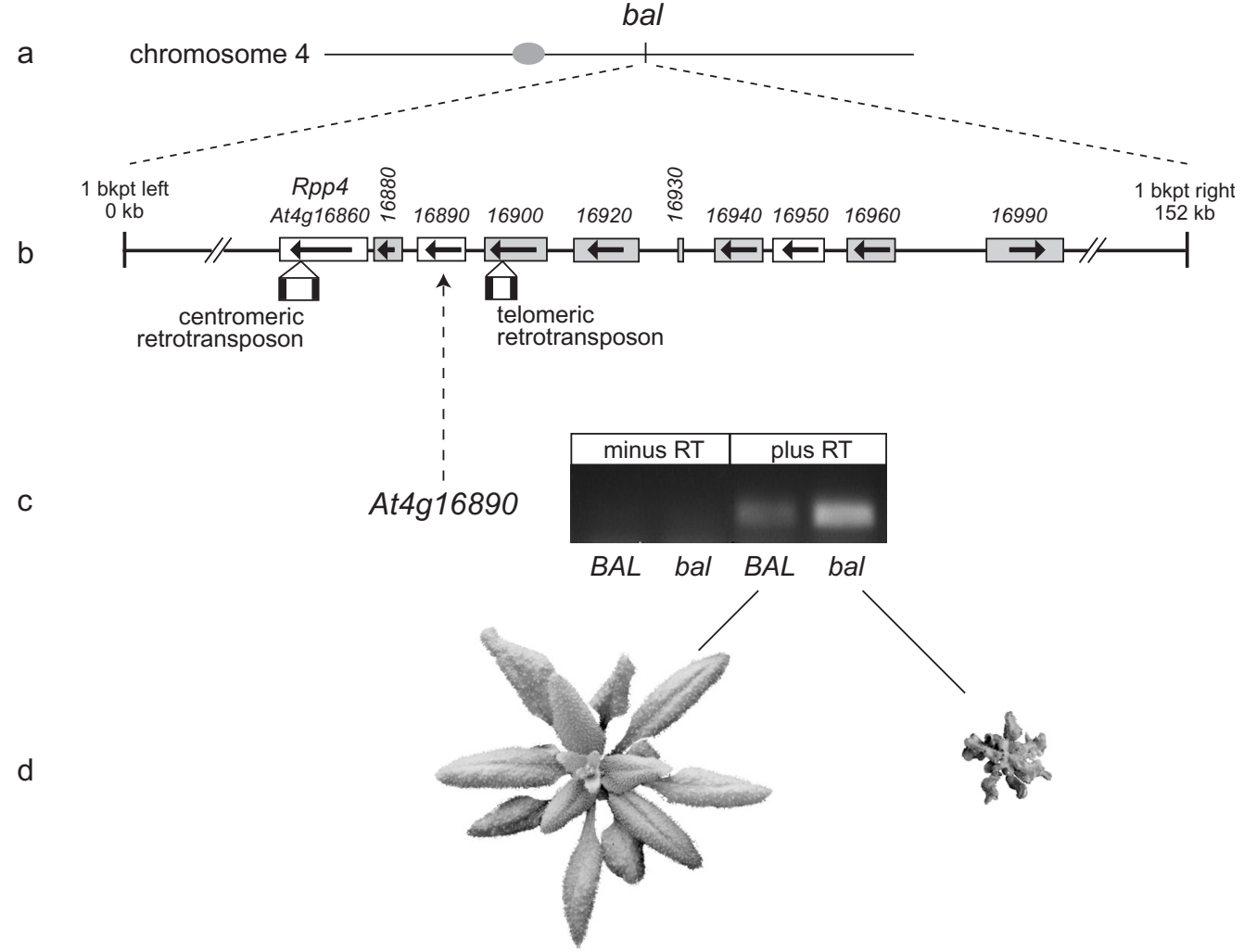

Figure 1. The $d d m l$-induced bal dwarfing syndrome is caused by misregulation of a pathogen resistance gene cluster. The bal allele maps to Arabidopsis chromosome $4(a)$ in a 152-kb interval $(b)$ containing a cluster of ten $R$ genes or gene fragments and transposable elements (in strain Columbia). The direction of gene transcription is indicated by the arrow. Open boxes, functional or potentially functional genes; gray boxes, genes predicted to be nonfunctional. (c) A reverse transcriptase polymerase chain reaction (RT-PCR) experiment (loading controls not shown) demonstrating overexpression of At4g16890 in bal plants. (d) The phenotypes of a wild-type (BAL) individual and bal dwarf (strain Columbia). 
Support for the hypothesis that the bal syndrome is caused by an epigenetic allele comes from the unusual metastability of the bal alteration. We have not observed reversion or instability of the bal defect under standard greenhouse conditions, suggesting that the bal allele is very stable. Yet, attempts to isolate suppressor mutations by mutagenizing a bal homozygote yielded wild-type $B A L$ revertants at a frequency approaching $10 \%$ in the first generation after mutagenesis with ethylmethanesulfonate (EMS) (Stokes et al. 2002; H. Yi and E.J. Richards, unpubl.). This frequency is orders of magnitude higher than that expected from reversion of point mutations. No rearrangements or transposition events were observed in the locus, arguing against high-frequency genetic reversion mechanisms. As expected, reversion to the $B A L$ state is accompanied by the loss of $A t 4 g 16890$ overexpression.

The chromosome $4 R$-gene cluster acts as an island of heterochromatin and appears to be a hot spot for epigenetic variation. At least two other metastable alterations mapping to or near the same chromosome $4 R$ gene cluster have been reported. Both alterations, ssil (Shah et al. 1999) and cprl (Bowling et al. 1994), display (1) constitutive pathogen signaling and dwarfing, (2) metastability, and (3) overexpression of at least one $R$ gene related to At4g16890 (Stokes and Richards 2002; Stokes et al. 2002; Yang and Hua 2004). One objective of our current efforts is to understand the molecular mechanisms that underlie the formation and metastability of bal and related alterations as a model for the types of epigenetic mechanisms that exercise control in complex genomic regions typical of eukaryotic genomes.

Interestingly, both ssil and cprl were generated from EMS mutageneses (Bowling et al. 1994; Shah et al. 1999). Two of the best studied Arabidopsis epigenetic alleles - hypomethylated fwa flowering time alleles (Soppe et al. 2000) and hypermethylated sup flower development alleles (Jacobsen and Meyerowitz 1997)—were also isolated from screens of chemically mutagenized plants, demonstrating that traditional mutagenesis protocols can generate both genetic and epigenetic alleles.

\section{NATURAL EPIGENETIC VARIATION}

If it is well established that epigenetic variation can be generated at a high frequency in plants in response to traditional mutageneses or as a consequence of pharmacological or genetic manipulation of epigenetic modifications such as DNA methylation, the relevance of analogous epigenetic variants in natural plant populations is less clear. One striking example of an epigenetic variant in a wild population was described by Enrico Coen and colleagues in 1999 - a variant form of toadflax (in the genus Linaria) with radially rather than bilaterally symmetric flowers (Cubas et al. 1999). This morphologically distinct Linaria variant was recognized by Linnaeus over 250 years earlier. The mechanistic basis of the alternate forms in flower morphology is epigenetic silencing, associated with DNA hypermethylation, of the Linaria CYCLOIDEA homolog, Lcyc.
Our lab began an approach to study the prevalence and stability of epigenetic variation among natural accessions of Arabidopsis. These accessions are laboratory lines propagated from seeds originally collected from throughout the natural range of Arabidopsis and are often referred to as "ecotypes," suggesting that they represent different genotypes fine-tuned to specific ecological habitats. These lines capture a wide variety of variation that has been exploited as a source of new genetic alleles (AlonsoBlanco and Koornneef 2000). We asked the simple question, "How much epigenetic variation in the form of differential cytosine methylation is found among these accessions?" (also see Cervera et al. 2002). In our initial study (Riddle and Richards 2002), we found that a number of genomic sequences were invariably highly methylated, such as the 180-base pair (bp) centromeric repeats and the pericentromeric Athila transposable element. However, we found certain genomic loci were differentially methylated among the accessions surveyed. Particularly striking was the variation in DNA methylation on the highly repeated ribosomal RNA genes found in large arrays at the top of chromosome 2 and chromosome 4, the so-called nucleolus organizer regions (NORs) (Fig. 2).

The different NOR methylation phenotypes characteristic of the different accessions result from a combination of sources. Two lines of evidence indicate that differential NOR methylation is partially due to genetic variation (Riddle and Richards 2002). First, the number of rRNA genes strongly correlates with total NOR methylation content, in keeping with previous observations suggest-

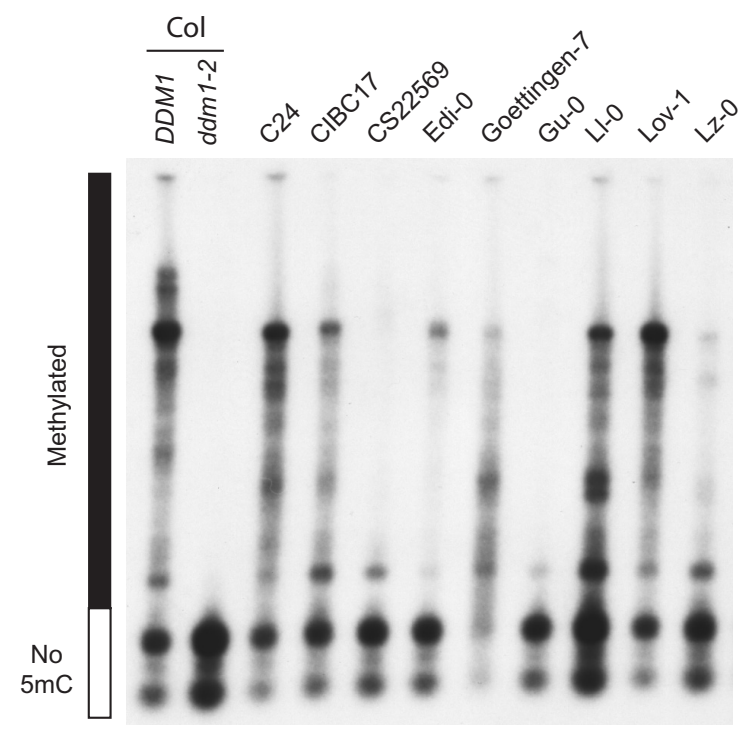

Figure 2. Natural variation in rRNA gene methylation among different Arabidopsis accessions. Southern blot assay to monitor genomic cytosine methylation among different Arabidopsis strains (http://walnut.usc.edu/2010.html) and controls (Columbia wild-type $=\mathrm{Col} D D M 1$; plus a $d d m 1-2$ homozygote in a Columbia background). The hybridization signal indicates the extent of methylation in the rRNA gene clusters; unmethylated fragments are cleaved by the methylation-sensitive endonuclease HpaII to small fragments (open box), whereas methylated genomic fragments remain uncleaved or partially cleaved and migrate as larger fragments (solid box). 
ing that excess rRNA gene repeats are archived in a silenced, methylated state. A second line of evidence comes from quantitative trait loci (QTL) analysis using recombinant inbred (RI) lines generated from crosses between the high NOR methylation accession Ler and the low NOR methylation accession Cvi. At least two significant trans-acting QTL influence the variation of NOR methylation among the different RI lines. But total NOR methylation is not under strict genetic control; QTL analysis and supporting studies demonstrate that the inheritance and maintenance of differential NOR methylation states are the most important factors explaining the variation in NOR methylation among the RI lines. These findings suggest that the unique NOR methylation content characteristic of different Arabidopsis accessions results from a combination of genetic factors (i.e., rRNA gene number and trans-acting modifiers) pushing against the inertia of the parental NOR methylation state.

These results provide some insight into the forces and dynamics that shape cytosine methylation patterns in natural populations. In the short timescale represented by the transmission genetic experiments described above (i.e., a handful of generations), epigenetic inheritance is paramount in defining genomic cytosine methylation patterns. Over longer time frames, genetic control of epigenetic modification is expected to play the dominant role. Nonetheless, the importance of stochastic changes that shift cytosine methylation patterns cannot be discounted. For example, there is precedent for abiotic stresses (e.g., temperature; Sherman and Talbert 2002) or biotic stresses (e.g., pathogen infection; Guseinov and Vanyushin 1975) altering cytosine methylation levels. Such changes would establish a new methylation default state that would be inherited over time because of the self-propagating nature of epigenetic codes. Thus, epigenetic variation need not march in lockstep with genetic variation at loci that are targets of epigenetic modification or loci that encode the machinery that executes or guides this modification.

\section{CONCLUSIONS}

The modes of induction, meiotic transmission, and genetic behavior of alternative cytosine methylation alleles in Arabidopsis suggest that epigenetic alleles will be frequently encountered in plant genetics and often mistaken for genetic mutations. The larger questions concerning the relevance of epigenetic variation in natural populations and in an evolutionary context persist. The semi-independent nature of epigenetic variation - the possibility of formation and transmission of epigenetic alleles independent of genetic variation controlling these eventssuggests a plausible role for epigenetic variation in evolution. A single genotype can express a range of phenotypes based on alternative epigenotypes. This mechanism incorporates an element beyond that implied by phenotypic plasticity because epigenetic information can be inherited. Selection for one alternative epigenotype may serve as an intermediate bridge to a greater stabilization of an adaptive phenotypic outcome by genetic variation.
This chain of events is a type of "genetic assimilation" mechanism, a process through which apparently environmentally induced phenotypic variation can be inherited. As originally formulated and demonstrated by Waddington $(1942,1953)$, genetic assimilation involves the selection of cryptic genetic variation uncovered by environmental stress. Selection for stress-induced phenotypes leads to the accumulation of genetic alleles, the effects of which are now exposed in the new environment, that favor the expression of the selected phenotype. After several generations of selection the variants express the abnormal phenotype in the absence of the inducing environment. Inherited epigenetic variation might play two roles in the genetic assimilation of adaptive phenotypes. In one scenario, selection would initially act on alternative heritable epigenetic states rather than on newly exposed genetic variation. Subsequent selection for genetic alleles that buttress or supplant the epigenetic changes would complete the assimilation. Alternatively, selection may be operating on both genetic and epigenetic variation (Ruden et al. 2003; Sollars et al. 2003), with inherited epigenetic variation stabilizing the selected phenotype in transition to complete genetic assimilation.

\section{ACKNOWLEDGMENTS}

We thank Justin Rincker for comments on the manuscript. Research in our group has been supported by grants from the National Science Foundation (DBI9975930, MCB-9985348, and MCB-0321990) and the Ministry of Agriculture, Forestry and Fisheries, Japan.

\section{REFERENCES}

Alonso-Blanco C. and Koornneef M. 2000. Naturally occurring variation in Arabidopsis: An underexploited resource for plant genetics. Trends Plant Sci. 5: 22.

Bowling S.A., Guo A., Cao H., Gordon A.S., Klessig D.F., and Dong X. 1994. A mutation in Arabidopsis that leads to constitutive expression of systemic acquired resistance. Plant Cell 6: 1845.

Cervera M.T., Ruiz-Garcia L., and Martinez-Zapater J.M. 2002. Analysis of DNA methylation in Arabidopsis thaliana based on methylation-sensitive AFLP markers. Mol. Genet. Genomics 268: 543 .

Chandler V.L. and Stam M. 2004. Chromatin conversations Mechanisms and implications of paramutation. Nat. Rev. Genet. 5: 532.

Cubas P., Vincent C., and Coen E. 1999. An epigenetic mutation responsible for natural variation in floral symmetry. Nature 401: 157.

Dangl J.L. and Jones J.D. 2001. Plant pathogens and integrated defence responses to infection. Nature 411: 826 .

Guseinov V.A. and Vanyushin B.F. 1975. Content and localisation of 5-methylcytosine in DNA of healthy and wilt-infected cotton plants. Biochim. Biophys. Acta 395: 229.

Holliday R. and Ho T. 1990. Evidence for allelic exclusion in Chinese hamster ovary cells. New Biol. 2: 719.

Jacobsen S.E. and Meyerowitz E.M. 1997. Hypermethylated SUPERMAN epigenetic alleles in Arabidopsis. Science 277: 1100 .

Jeddeloh J.A., Stokes T.L., and Richards E.J. 1999. Maintenance of genomic methylation requires a SWI2/SNF2-like protein. Nat. Genet. 22: 94.

Kakutani T. 1997. Genetic characterization of late-flowering traits induced by DNA hypomethylation mutation in Arabidopsis thaliana. Plant J. 12: 1447. 
Kakutani T., Munakata K., Richards E.J., and Hirochika H. 1999. Meiotically and mitotically stable inheritance of DNA hypomethylation induced by ddm 1 mutation of Arabidopsis thaliana. Genetics 151: 831.

Kakutani T., Jeddeloh J.A., Flowers S.K., Munakata K., and Richards E.J. 1996. Developmental abnormalities and epimutations associated with DNA hypomethylation mutations. Proc. Natl. Acad. Sci. 93: 12406.

Kankel M.W., Ramsey D.E., Stokes T.L., Flowers S.K., Haag J.R., Jeddeloh J.A., Riddle N.C., Verbsky M.L., and Richards E.J. 2003. Arabidopsis MET1 cytosine methyltransferase mutants. Genetics 163: 1109.

Li E., Bestor T.H., and Jaenisch R. 1992. Targeted mutation of the DNA methyltransferase gene results in embryonic lethality. Cell 69: 915.

Macleod D., Clark V.H., and Bird A. 1999. Absence of genome-wide changes in DNA methylation during development of the zebrafish. Nat. Genet. 23: 139.

Maynard Smith J. 1990. Models of a dual inheritance system. $J$. Theor. Biol. 143: 41.

McClintock B. 1952. Chromosome organization and genic expression. Cold Spring Harbor Symp. Quant. Biol. 16: 13.

Morgan H.D., Sutherland H.G., Martin D.I., and Whitelaw E. 1999. Epigenetic inheritance at the agouti locus in the mouse. Nat. Genet. 23: 314.

Pikaard C.S. 2000. The epigenetics of nucleolar dominance. Trends Genet. 16: 495.

Plath K., Mlynarczyk-Evans S., Nusinow D.A., and Panning B. 2002. Xist RNA and the mechanism of X chromosome inactivation. Annu. Rev. Genet. 36: 233.

Rakyan V.K., Chong S., Champ M.E., Cuthbert P.C., Morgan H.D., Luu K.V., and Whitelaw E. 2003. Transgenerational inheritance of epigenetic states at the murine Axin $(\mathrm{Fu})$ allele occurs after maternal and paternal transmission. Proc. Natl. Acad. Sci. 100: 2538.

Reik W., Dean W., and Walter J. 2001. Epigenetic reprogramming in mammalian development. Science 293: 1089.

Rice J.C. and Allis C.D. 2001. Code of silence. Nature 414: 258.

Richards E.J. and Elgin S.C. 2002. Epigenetic codes for heterochromatin formation and silencing: Rounding up the usual suspects. Cell 108: 489.

Riddle N.C. and Richards E.J. 2002. The control of natural variation in cytosine methylation in Arabidopsis. Genetics 162: 355 .
Ruden D.M., Garfinkel M.D., Sollars V.E., and Lu X. 2003. Waddington's widget: Hsp90 and the inheritance of acquired characters. Semin. Cell Dev. Biol. 14: 301.

Russo V.E.A., Martienssen R.A., and Riggs A.D., eds. 1996. Epigenetic mechanisms of gene regulation. Cold Spring Harbor Laboratory Press, Cold Spring Harbor, New York.

Santos F., Hendrich B., Reik W., and Dean W. 2002. Dynamic reprogramming of DNA methylation in the early mouse embryo. Dev. Biol. 241: 172 .

Shah J., Kachroo P., and Klessig D.F. 1999. The Arabidopsis ssil mutation restores pathogenesis-related gene expression in npr1 plants and renders defensin gene expression salicylic acid dependent. Plant Cell 11: 191.

Sherman J.D. and Talbert L.E. 2002. Vernalization-induced changes of the DNA methylation pattern in winter wheat. Genome 45: 253.

Silva A.J. and White R. 1988. Inheritance of allelic blueprints for methylation patterns. Cell 54: 145

Sollars V., Lu X., Xiao L., Wang X., Garfinkel M.D., and Ruden D.M. 2003. Evidence for an epigenetic mechanism by which Hsp90 acts as a capacitor for morphological evolution. Nat. Genet. 33: 70.

Soppe W.J., Jacobsen S.E., Alonso-Blanco C., Jackson J.P., Kakutani T., Koornneef M., and Peeters A.J. 2000. The late flowering phenotype of fwa mutants is caused by gain-offunction epigenetic alleles of a homeodomain gene. Mol. Cell 6: 791.

Stokes T.L. and Richards E.J. 2002. Induced instability of two Arabidopsis constitutive pathogen-response alleles. Proc. Natl. Acad. Sci. 99: 7792.

Stokes T.L., Kunkel B.N., and Richards E.J. 2002. Epigenetic variation in Arabidopsis disease resistance. Genes Dev. 16: 171.

Vongs A., Kakutani T., Martienssen R.A., and Richards E.J. 1993. Arabidopsis thaliana DNA methylation mutants. Science 260: 1926.

Waddington C.H. 1939. An introduction to modern genetics. Allen and Unwin, London.

1942. Canalization of development and the inheritance of acquired characters. Nature 150: 563.

1953. Genetic assimilation of an acquired character. Evolution 7: 118.

Yang S. and Hua J. 2004. A haplotype-specific Resistance gene regulated by BONZAI1 mediates temperature-dependent growth control in Arabidopsis. Plant Cell 16: 1060. 


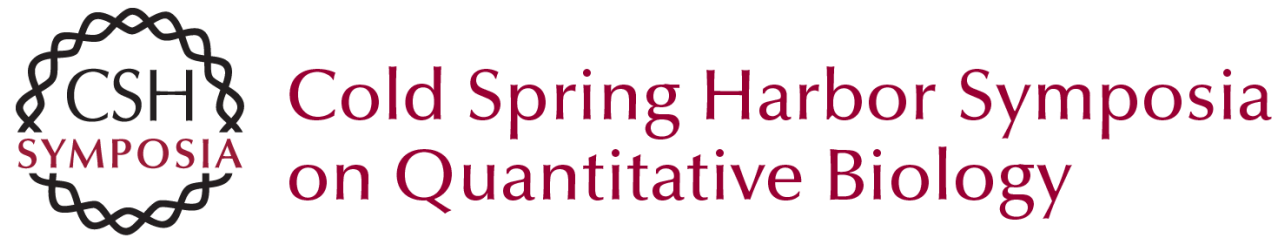

\section{Induced and Natural Epigenetic Variation}

H. YI, N.C. RIDDLE, T.L. STOKES, et al.

Cold Spring Harb Symp Quant Biol 2004 69: 155-160

Access the most recent version at doi:10.1101/sqb.2004.69.155

References This article cites 39 articles, 13 of which can be accessed free at: http://symposium.cshlp.org/content/69/155.full.htmI\#ref-list-1

\section{License}

Email Alerting Receive free email alerts when new articles cite this article - sign up in Service the box at the top right corner of the article or click here. 